\title{
Moments of inertia of relativistic magnetized stars
}

\author{
K. Konno \\ Department of Physics, Hiroshima University, Higashi-Hiroshima 739-8526, Japan \\ Received 3 October 2000 / Accepted 6 April 200

\begin{abstract}
We consider principal moments of inertia of axisymmetric, magnetically deformed stars in the context of general relativity. The general expression for the moment of inertia with respect to the symmetric axis is obtained. The numerical estimates are derived for several polytropic stellar models. We find that the values of the principal moments of inertia are modified by a factor of 2 at most from Newtonian estimates.
\end{abstract}

Key words. stars: pulsars: general - stars: magnetic fields - stars: rotation - relativity

\section{Introduction}

Various features of pulsars have been disclosed with growing observational data. Most pulsars have stable pulse shapes, and spin down steadily with typical time-scales of the order of several Myr (see e.g. Mészáros 1992). However, deviations from linear spin-down of trends have also been observed on shorter time-scales in some pulsars. These deviations convey information about the surroundings, internal structure and dynamics of neutron stars. For example, the presence of glitches (see e.g. Shemar \& Lyne 1996; Wang et al. 2000) indicates that two or more poorly coupled components coexist in the internal structure of the stars, while timing noise (see e.g. D'Alessandro 1995) is believed to be due to random movements of the fluid in pulsars. Besides these short time-scale instabilities, the spin-down features indicating the precession due to stellar deformation have also been reported. Two anomalous X-ray pulsars (AXPs), 1E1048.1-5937 and 1E2259+586, were observed to indicate irregular spin-down (see e.g. Mereghetti 1995; Baykal \& Swank 1996; Baykal et al. 1998; Oosterbroek et al. 1998). AXPs are a subclass of X-ray pulsars with periods in a very narrow range (6-12 s) and period derivatives in the range $\left(10^{-12}-10^{-11} \mathrm{~s} \mathrm{~s}^{-1}\right.$ ) (see e.g. Mereghetti \& Stella 1995), and which are most likely candidates of magnetars (Duncan \& Thompson 1992; Thompson \& Duncan 1993, 1995, 1996) along with soft gamma repeaters (SGRs) (see e.g. Kouveliotou et al. 1998, 1999). As discussed by Melatos (1999), the wobbles in their spin-down rates may be interpreted by an effect called radiative precession (Melatos 2000), which is related to an oscillating component of electromagnetic torque. Another precessing pulsar PSR B1828-11 (Stairs et al. 2000) was reported very recently. This gives the very clear observation of free preces-

\footnotetext{
* e-mail: konno@theo.phys.sci.hiroshima-u.ac.jp
}

sion of an isolated neutron star. These deformed, precessing objects are analyzed by solving the Euler equation of motion in the form $I_{i j} \mathrm{~d} \Omega^{j} / \mathrm{d} t-\varepsilon_{i j k} I^{j l} \Omega^{k} \Omega_{l}=N_{i}$, where $I_{i j}$ is the inertia tensor, $\Omega^{i}$ is the angular velocity and $N_{i}$ is the torque acting on the object. When we take into account the electromagnetic torque by a rotating magnetic dipole (Davis \& Goldstein 1970; Goldreich 1970), the above-mentioned radiative precession can be found for magnetically deformed stars (Melatos 1999; 2000). Furthermore, since magnetically deformed, rotating stars emit gravitational waves, we can consider the gravitational radiation reaction torque (Bertotti \& Anile 1973; Cutler \& Jones 2000). The gravitational backreaction damps the wobbles on a time-scale proportional to $\left[\left(I_{z}-I_{x}\right)^{2} / I_{x}\right]^{-1}$ for wobbling, axisymmetric rigid bodies, where $I_{i}$ is the principal moments of inertia. Thus, the moments of inertia play a significant role in the analyses of pulsar precession. The estimates of the moments of inertia have been done in the context of Newtonian gravity so far. However, neutron stars are fully general relativistic objects, and it is important to take into account general relativistic effects. Therefore, we now discuss the principal moments of inertia of magnetically deformed stars in the context of general relativity.

In Newtonian gravity, once we have the mass distribution of an object $\rho(\boldsymbol{x})$, the moment of inertia with respect to any axis can be calculated from $I=\int \rho(\boldsymbol{x}) \chi^{2} \mathrm{~d}^{3} x$, where $\chi$ denotes the length from the axis. In general relativity, only for axisymmetric objects, the moment of inertia with respect to the symmetric axis can be well defined. For this definition, we need slow rotation of the objects. The slow rotation ensures that the angular momentum $J$ is linearly related to the angular velocity $\Omega$, i.e. $J=I \Omega$. Here, $I$ defines the general relativistic version of the moment of inertia. The moments of inertia of 
relativistic, spherically symmetric stars were discussed by Hartle (1967) and Chandrasekhar \& Millar (1974). The approximate expression valid for various realistic equations of state was also derived by Ravenhall \& Pethick (1994).

In this paper, we discuss the principal moments of inertia of magnetically deformed stars which are axisymmetric with respect to the magnetic axis. Deformation of relativistic magnetized stars was studied both in the numerical approach (Bonazzola et al. 1993; Bocquet et al. 1995) and in the analytic approach (Konno et al. 1999). Based on the latter, analytic approach developed by Konno et al. (1999), we develop the subsequent discussion. In the previous work (Konno et al. 1999), stellar magnetic fields were regarded as corrections to non-rotating, spherical stars, and the magnetic deformation of stars was formulated using a perturbative approach. As mentioned above, we now take into account the slow rotation of the deformed stars on the symmetric axis to define the moment of inertia. The formulation of this configuration is given in Sect. 2. The general expression for the principal moment of inertia with respect to the symmetric axis is also derived. The numerical estimates are obtained for several stellar models in Sect. 3. In Sect. 4, we discuss the other principal moments of inertia. Finally, we give our conclusions in Sect. 5. Throughout this paper, we use units in which $c=G=1$.

\section{Magnetically deformed rotating stars}

We consider a slowly rotating star which is subject to quadrupole deformation due to a dipole magnetic field. As described by Konno et al. (1999), magnetic fields can be treated as corrections to a background, spherically symmetric star, i.e. $B \sim O\left(\varepsilon_{\mathrm{B}}\right)$. We now assume that the star slowly rotates on the magnetic axis with a uniform angular velocity $\Omega \sim O\left(\varepsilon_{\Omega}\right)$, which is also regarded as a perturbation. In this paper, we take into account the rotational corrections up to first order in $\varepsilon_{\Omega}$. The metric describing such a star can be given by

$$
\begin{aligned}
\mathrm{d} s^{2}= & -\mathrm{e}^{\nu(r)}\left[1+2\left(h_{0}(r)+h_{2}(r) P_{2}(\cos \theta)\right)\right] \mathrm{d} t^{2} \\
& +\mathrm{e}^{\lambda(r)}\left[1+\frac{2 \mathrm{e}^{\lambda(r)}}{r}\left(m_{0}(r)+m_{2}(r) P_{2}(\cos \theta)\right)\right] \mathrm{d} r^{2} \\
& +r^{2}\left(1+2 k_{2}(r) P_{2}(\cos \theta)\right) \mathrm{d} \theta^{2} \\
& +r^{2} \sin ^{2} \theta\left(1+2 k_{2}(r) P_{2}(\cos \theta)\right) \\
& \times\left[\mathrm{d} \phi-\left(W_{1}(r)-\frac{W_{3}(r)}{\sin \theta} \frac{\mathrm{d} P_{3}(\cos \theta)}{d \theta}\right) \mathrm{d} t\right]^{2},
\end{aligned}
$$

where $P_{l}$ is the Legendre polynomial of order $l$, and $\nu$ and $\lambda$ describe a background star. The corrections $h_{0}, h_{2}$, $m_{0}, m_{2}$ and $k_{2}$ of second order in $\varepsilon_{\mathrm{B}}$ correspond to deviation from the spherical shape. If we replace $W_{1}$ and $W_{3}$ with zero in Eq. (1), this expression reduces to the metric already investigated by Konno et al. (1999). The newly appearing functions $W_{1}$ and $W_{3}$ lead to frame dragging due to the magnetically deformed, rotating star. In order to involve the effect of deformation, we let these functions include corrections up to order $\varepsilon_{\Omega} \varepsilon_{\mathrm{B}}^{2}$. Showing the order explicitly, we can write $W_{1}$ and $W_{3}$ in the forms

$$
\begin{aligned}
& W_{1}=\omega+W_{1}^{(2)}, \\
& W_{3}=W_{3}^{(2)},
\end{aligned}
$$

where $\omega \sim O\left(\varepsilon_{\Omega}\right)$ and $\left(W_{1}^{(2)}, W_{3}^{(2)}\right) \sim O\left(\varepsilon_{\Omega} \varepsilon_{\mathrm{B}}^{2}\right)$. The form of the terms including $W_{1}$ and $W_{3}$ in Eq. (1) corresponds to quadrupole deformation of the star. These functions are analogous to the rotational corrections up to third order in $\varepsilon_{\Omega}$ (Quintana 1976; Kojima \& Hosonuma 1999).

The stress-energy tensor of the star which is composed of a perfect fluid endowed with a dipole magnetic field has the form

$$
\begin{aligned}
T_{\nu}^{\mu}= & (\rho+p) u^{\mu} u_{\nu}+p \delta_{\nu}^{\mu} \\
& +\frac{1}{4 \pi}\left(F^{\mu \lambda} F_{\nu \lambda}-\frac{1}{4} F^{\sigma \lambda} F_{\sigma \lambda} \delta^{\mu}{ }_{\nu}\right)
\end{aligned}
$$

where the density $\rho$ and the pressure $p$ are expanded as

$\rho=\rho_{0}(r)+\left(\rho_{20}(r)+\rho_{22}(r) P_{2}\right)$
$p=p_{0}(r)+\left(p_{20}(r)+p_{22}(r) P_{2}\right)$.

Here, $\rho_{20}, \rho_{22}, p_{20}$ and $p_{22}$ are second-order quantities in $\varepsilon_{\mathrm{B}}$. The non-vanishing components of the 4 -velocity $u^{\mu}$ are given by

$$
u^{t}=\mathrm{e}^{-\frac{\nu}{2}}\left[1-\left(h_{0}+h_{2} P_{2}\right)\right], \quad u^{\phi}=\Omega u^{t} .
$$

Furthermore, the Faraday tensor $F_{\mu \nu}$ can be given by the 4-potential $A_{\mu}$ written as (Muslimov \& Tsygan 1986; Konno \& Kojima 2000)

$$
A_{\mu}=\left(a_{t 0}(r)+a_{t 2}(r) P_{2}, 0,0, a_{\phi}(r) \sin \theta \frac{\mathrm{d} P_{1}}{\mathrm{~d} \theta}\right),
$$

where the $\phi$-component corresponds to a dipole magnetic field, and the $t$-component denotes the electric field induced by stellar rotation. Therefore, we have $a_{\phi} \sim O\left(\varepsilon_{\mathrm{B}}\right)$ and $\left(a_{t 0}, a_{t 2}\right) \sim O\left(\varepsilon_{\Omega} \varepsilon_{\mathrm{B}}\right)$.

The above-mentioned functions except $W_{1}$ and $W_{3}$ were investigated in detail by Konno et al. (1999) and Konno \& Kojima (2000) (see Appendix A for a brief summary). The differential equations which $W_{1}$ and $W_{3}$ obey can be obtained from the $(t \phi)$-component of the Einstein equation,

$\frac{1}{r^{4}} \frac{\mathrm{d}}{\mathrm{d} r}\left[r^{4} j \frac{\mathrm{d} \bar{W}_{1}}{\mathrm{~d} r}\right]+\frac{4}{r} \frac{\mathrm{d} j}{\mathrm{~d} r} \bar{W}_{1}=-j\left(S_{1}-\frac{S_{2}}{5}\right)$,

$\frac{1}{r^{4}} \frac{\mathrm{d}}{\mathrm{d} r}\left[r^{4} j \frac{\mathrm{d} W_{3}}{\mathrm{~d} r}\right]+\left(\frac{4}{r} \frac{\mathrm{d} j}{\mathrm{~d} r}-\frac{10}{r^{2}} \mathrm{e}^{\frac{\lambda-\nu}{2}}\right) W_{3}=j \frac{S_{2}}{5}$,

where $j$ and $\bar{W}_{1}$ are defined, respectively, as

$j=\mathrm{e}^{-\frac{\nu+\lambda}{2}}$

$\bar{W}_{1}=\Omega-W_{1}=\varpi-W_{1}^{(2)} \quad(\varpi=\Omega-\omega)$. 
These differential equations are solved order by order. The differential equation for $\varpi$ of order $\varepsilon_{\Omega}$, in which both $S_{1}$ and $S_{2}$ vanish, was solved by Hartle (1967). We are now interested in the differential equations of order $\varepsilon_{\Omega} \varepsilon_{\mathrm{B}}^{2}$. The functions $S_{1}$ and $S_{2}$ appearing in the source terms in Eqs. (9) and (10), in general, include $W_{1}$ and $W_{3}$. However, when we consider $S_{1}$ and $S_{2}$ up to the order of our interest, these functions include $\Omega$ and $\omega$ only as shown below. The source terms of order $\varepsilon_{\Omega} \varepsilon_{\mathrm{B}}^{2}$ are given by

$$
\begin{aligned}
S_{1}= & \varpi^{\prime}\left(-h_{0}-\frac{\mathrm{e}^{\lambda}}{r} m_{0}\right)^{\prime}-\frac{4}{r^{2}} \mathrm{e}^{\lambda}\left(\nu^{\prime}+\lambda^{\prime}\right) \varpi m_{0} \\
& -16 \pi \mathrm{e}^{\lambda} \varpi\left(\rho_{20}+p_{20}\right)+\frac{16 \mathrm{e}^{\lambda}}{3 r^{4}} a_{\phi}^{2} \omega \\
& +\frac{8}{3 r^{2}}\left(a_{\phi}^{\prime}\right)^{2} \omega+\frac{8 \mathrm{e}^{\lambda}}{r^{4}} a_{\phi} a_{t 2}-\frac{4}{r^{2}} a_{\phi}^{\prime} a_{t 0}^{\prime} \\
S_{2}= & \varpi^{\prime}\left(4 k_{2}-h_{2}-\frac{\mathrm{e}^{\lambda}}{r} m_{2}\right)^{\prime}-\frac{4}{r^{2}} \mathrm{e}^{\lambda}\left(\nu^{\prime}+\lambda^{\prime}\right) \varpi m_{2} \\
& -16 \pi \mathrm{e}^{\lambda} \varpi\left(\rho_{22}+p_{22}\right)+\frac{32 \mathrm{e}^{\lambda}}{3 r^{4}} a_{\phi}^{2} \omega \\
& -\frac{8}{3 r^{2}}\left(a_{\phi}^{\prime}\right)^{2} \omega+\frac{16 \mathrm{e}^{\lambda}}{r^{4}} a_{\phi} a_{t 2}-\frac{4}{r^{2}} a_{\phi}^{\prime} a_{t 2}^{\prime}
\end{aligned}
$$

where the prime here denotes differentiation with respect to $r$. These functions can be calculated by using the results for the density and pressure $\left(\rho_{20}, \rho_{22}, p_{20}, p_{22}\right)$, the metric functions $\left(h_{0}, h_{2}, m_{0}, m_{2}, k_{2}\right)$ and the potential functions $\left(a_{\phi}, a_{t 0}, a_{t 2}\right)$, which have been calculated by Konno et al. (1999) and Konno \& Kojima (2000).

The differential equations (9) and (10) can be solved numerically by imposing boundary and junction conditions. The boundary conditions are summarized as

$$
\begin{aligned}
& W_{1} \rightarrow \text { const }, \quad W_{3} \rightarrow 0 \quad \text { as } \quad r \rightarrow 0 \\
& W_{1}, W_{3} \rightarrow \frac{1}{r^{\beta}}(\beta \geq 3) \quad \text { as } \quad r \rightarrow \infty
\end{aligned}
$$

Furthermore, we impose the junction condition given by (O’Brien \& Synge 1952; see also Eqs. (A.10) and (A.11))

$$
\begin{aligned}
& \left.W_{a}\right|_{+\xi^{(0)}}=\left.W_{a}\right|_{-\xi^{(0)}}, \\
& \left.W_{a}^{\prime}\right|_{+\xi^{(0)}}=\left.W_{a}^{\prime}\right|_{-\xi^{(0)}},
\end{aligned}
$$

where $a$ takes 1 or 3 , and $\xi^{(0)}$ denotes the surface of the background star.

Before deriving numerical solutions, it is worthwhile investigating the behavior of $W_{1}$ and $W_{3}$ at large $r$ in detail using Eqs. (9), (10), (13) and (14), because this inspection gives the expressions for the angular momentum and the moment of inertia of the star.

At large $r$, we have

$\lambda, \nu \rightarrow 0, \quad$ i.e. $\quad j \rightarrow 1$.

From Eqs. (13) and (14), we derive the asymptotic behaviors

$S_{1} \propto \frac{1}{r^{8}}, \quad S_{2} \propto \frac{1}{r^{7}}$.
Using these expressions, from Eqs. (9), (10) and (16), we obtain

$W_{1}^{(2)} \propto \frac{1}{r^{3}}, \quad W_{3}^{(2)} \propto \frac{1}{r^{5}}$.

Hence, we can put

$\bar{W}_{1}=\Omega-\frac{2 J}{r^{3}}-W_{1 p}^{(2)}$,

where $J$ is the angular momentum of the deformed star, and $W_{1 p}^{(2)} \sim O\left(1 / r^{6}\right)$ is a function of order $\varepsilon_{\Omega} \varepsilon_{\mathrm{B}}^{2}$. We now integrate Eq. (9) after multiplying both sides by $r^{4}$. Using the above expression (22), we can obtain the general expression for the principal moment of inertia

$$
\begin{aligned}
I_{z}= & \frac{J}{\Omega} \\
= & -\frac{2}{3} \int_{0}^{R} r^{3} \frac{\mathrm{d} j}{\mathrm{~d} r} \frac{\varpi}{\Omega} \mathrm{d} r-\frac{R^{4}}{6 \Omega}\left(\frac{\mathrm{d} W_{1 p}^{(2)}}{\mathrm{d} r}\right)_{R} \\
& +\frac{2}{3} \int_{0}^{R} r^{3} \frac{\mathrm{d} j}{\mathrm{~d} r} \frac{W_{1}^{(2)}}{\Omega} \mathrm{d} r \\
& -\frac{1}{6 \Omega} \int_{0}^{R} r^{4} j\left(S_{1}-\frac{S_{2}}{5}\right) \mathrm{d} r,
\end{aligned}
$$

where $R$ is the radius of the background star. The moment of inertia of the background, spherically symmetric star $I^{(0)}$ is given by (Hartle 1967; Ravenhall \& Pethick 1994)

$I^{(0)}=-\frac{2}{3} \int_{0}^{R} r^{3} \frac{\mathrm{d} j}{\mathrm{~d} r} \frac{\varpi}{\Omega} \mathrm{d} r$.

Therefore, we obtain

$$
\begin{aligned}
I_{z}^{(2)}= & -\frac{R^{4}}{6 \Omega}\left(\frac{\mathrm{d} W_{1 p}^{(2)}}{\mathrm{d} r}\right)_{R}+\frac{2}{3} \int_{0}^{R} r^{3} \frac{\mathrm{d} j}{\mathrm{~d} r} \frac{W_{1}^{(2)}}{\Omega} \mathrm{d} r \\
& -\frac{1}{6 \Omega} \int_{0}^{R} r^{4} j\left(S_{1}-\frac{S_{2}}{5}\right) \mathrm{d} r
\end{aligned}
$$

where we have used the decomposition $I_{z}=I^{(0)}+I_{z}^{(2)}$. The magnetic modification of the principal moment of inertia $I_{z}^{(2)}$ is the second order quantity in $\varepsilon_{\mathrm{B}}$. The numerical estimates of $I_{z}^{(2)}$ for several stellar models are given in the next section.

\section{Numerical estimates of the moment of inertia}

Using the boundary and junction conditions mentioned in the last section, we can derive the numerical solutions for $W_{1}$ and $W_{3}$ for any stellar models. The numerical results for the magnetic correction of the principal moment of inertia $I_{z}^{(2)}$ can also be obtained by using Eq. (25). We now show the results of $I_{z}^{(2)}$ obtained for several polytropic stellar models. In these calculations, we adopted a different condition from that of the previous study (Konno et al. 1999), in which sequences with constant central density were investigated. In the current case, in order to clarify the correspondence between non-magnetized and magnetized stars having same mass, we use the condition in 


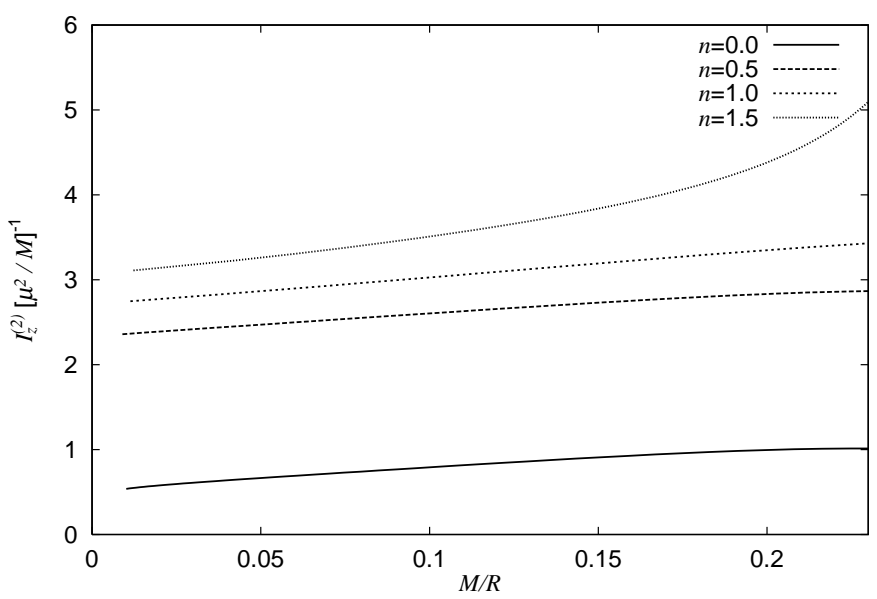

Fig. 1. The magnetic correction of the principal moment of inertia $I_{z}^{(2)}$ plotted as a function of $M / R$. The values are normalized by $\mu^{2} / M$, and the polytropic index is denoted by $n$.

which the total mass of the star does not change through the perturbative approach. This is also because the moment of inertia can be modified significantly by the mass shift rather than the magnetic deformation. In our formulation, this condition is accomplished by imposing the boundary condition that $m_{0}$ vanishes at infinity.

Figure 1 displays the magnetic correction $I_{z}^{(2)}$ as a function of the general-relativistic factor $M / R$. The values are normalized by the typical value $\mu^{2} / M$. As a simple example, we now discuss quadrupole deformation of a fluid body in the Newtonian limit. If the deformed body has constant density, which corresponds to $n=0$, then we can derive the result $I_{z}^{(2)}=10 \mu^{2} /(3 M)$ from the estimate of ellipticity $25 \mu^{2} /\left(2 M^{2} R^{2}\right)$ (Ferraro 1954; Konno et al. 2000). However, as seen in Fig. 1, our numerical result for $n=0$ is different from this simple estimate. This is because the perturbed star does not have constant effective density by the added perturbation, even though we assume the background star with constant density. This reason can be understood by seeing the differential equation for $m_{0}$, i.e. Eq. (A.3). The derivative of $m_{0}$ is related to the effective density including electromagnetic energy. In the case of $n=0$, although the first term on the right-hand side in Eq. (A.3) vanishes, the remaining terms do not vanish and are non-trivial functions. It follows that the effective density is not a constant. Thus, our results include the inertia of electromagnetic fields as well as mass. Therefore, our result shown in Fig. 1 cannot be compared with the above simple estimate.

Concentrating on the general relativistic effects on the magnetic correction $I_{z}^{(2)}$, we can find from Fig. 1 that the values of $I_{z}^{(2)}$ for each stellar model become large with the general relativistic factor $M / R$. The increments are $50 \%$ at most. However, the rates of increase may be neglected except for the case of $n=1.5$.

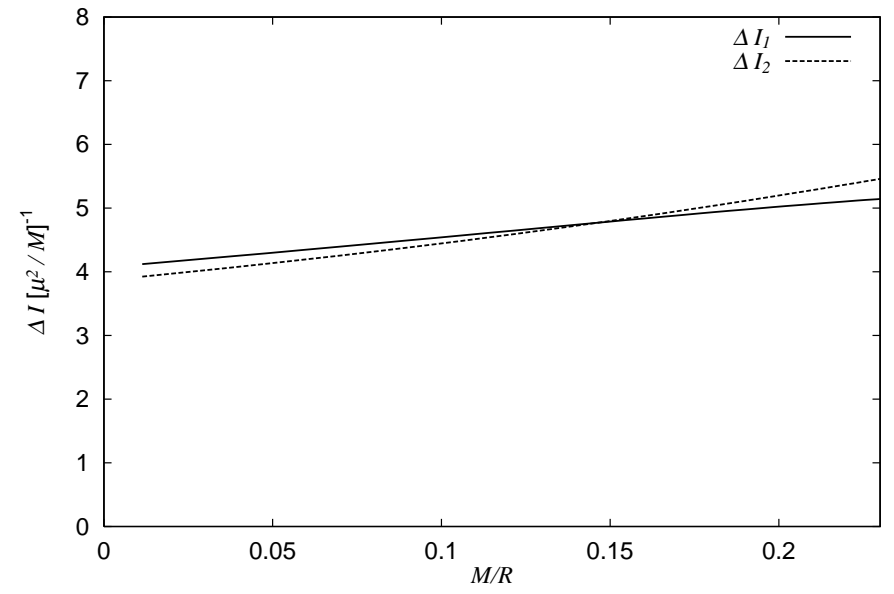

Fig. 2. Comparison between $\Delta I_{1}$ and $\Delta I_{2}$ for $n=1 . \Delta I_{1}$ and $\Delta I_{2}$ are normalized by $\mu^{2} / M$ and plotted as a function of $M / R$.

\section{The other components of moments of inertia}

Next, we discuss the other principal moments of inertia, i.e. $I_{x}^{(2)}$ and $I_{y}^{(2)}$. In the context of general relativity, the definition of these quantities is associated with a difficulty in the concept. As seen from Eqs. (22) and (23), the notion of moments of inertia is related to quantities at infinity (see also Geroch 1970; Hansen 1974). If we consider the rotation of the star on the $x$-axis or the $y$-axis, it produces the radiation of electromagnetic and gravitational fields. Thus, the exterior space-time is not stationary, but radiative. There is no rigorous way to define the moments of inertia in such a radiative space-time. The concept of $I_{x}^{(2)}$ and $I_{y}^{(2)}$ itself may be meaningless to a considerable extent.

Therefore, only approximate expressions are available. It would be useful to extend the Newtonian expressions, since it seems that most Newtonian features survive for neutron stars specified by the general relativistic factor $M / R \sim 0.2$. We cannot prove the validity mathematically, but will test some empirical relations. For this purpose, let us recall relations using the principal moments of inertia and the ellipticity which hold for an incompressible fluid in the context of Newtonian gravity. These are helpful in seeking the other principal moments of inertia. First, if the fluid body is subject to quadrupole deformation, then the relation between the principal moments of inertia exists

$I_{x}^{(2)}=I_{y}^{(2)}=-\frac{1}{2} I_{z}^{(2)}$.

Using this relation, the difference between the principal moments of inertia $I_{z}^{(2)}$ and $I_{x}^{(2)}$ is given by

$\Delta I_{1}=I_{z}^{(2)}-I_{x}^{(2)}=\frac{3}{2} I_{z}^{(2)}$.

This difference also can be expressed by using the ellipticity, which is defined by the difference between the equatorial radius and the polar radius (see e.g. 
Chandrasekhar \& Miller 1974; Konno et al. 1999; Konno et al. 2000),

$\Delta I_{2}=I^{(0)} \times($ ellipticity $)$.

Thus, the difference is derived by the two methods. There is no reason that the two expressions should coincide, but empirically the agreement is good. Note that these expressions (27) and (28) should coincide exactly in this case.

If we assume that the relation (26) is applicable to general relativistic cases, it seems that we should simply apply relation (26) to the results shown in Fig. 1 and derive $I_{x}^{(2)}$ and $I_{y}^{(2)}$. However, in order to utilize this relation, the magnetic corrections must be purely quadrupole contributions. As seen from the metric (1), the magnetic deformation includes monopole parts as well as quadrupole parts. Thus, our results in Fig. 1 also include monopole parts. Hence, we have to subtract the monopole contributions from $I_{z}^{(2)}$ to derive $I_{x}^{(2)}$ and $I_{y}^{(2)}$. It is, nevertheless, not so easy to extract monopole contributions accurately from these magnetically deformed stars.

However, there is no guarantee that all the results in Fig. 1 include monopole contributions dominantly. If there is a case in which the monopole part can be neglected, then we can estimate $I_{x}^{(2)}$ and $I_{y}^{(2)}$, by assuming that the relation (26) holds approximately. That case would also provide some extrapolation for general relativistic effects on $I_{x}^{(2)}$ and $I_{y}^{(2)}$ in the other cases. In order to seek such a case, we now compare $\Delta I_{1}$ derived by simply using the results in Fig. 1 with $\Delta I_{2}$ calculated from the ellipticity, which was already estimated for several stellar models (Konno et al. 2000). In the case that $\Delta I_{1}$ is almost consistent with $\Delta I_{2}$ about the values and tendency, it seems that the monopole part can be neglected, and we may use the relation (26). From the comparison, we find that $\Delta I_{1}$ is almost consistent with $\Delta I_{2}$ in the case of $n=1$. Figure 2 displays the comparison between $\Delta I_{1}$ and $\Delta I_{2}$ in this stellar model. The two curves coincide within $10 \%$. Hence, we can estimate $I_{x}^{(2)}$ and $I_{y}^{(2)}$ using Eq. (26) in this stellar model. Since the values of $I_{x}^{(2)}$ and $I_{y}^{(2)}$ are simply given by multiplying $I_{z}^{(2)}$ by a factor of $-1 / 2$, the changes of the absolute values of $I_{x}^{(2)}$ and $I_{y}^{(2)}$ due to general relativistic effects are specified by the same factor as in the case of $I_{z}^{(2)}$ (see Fig. 3). Consequently, we derive very similar result to that of $I_{z}^{(2)}$. However, $I_{x}^{(2)}$ and $I_{y}^{(2)}$ decrease with the general relativistic factor $M / R$, while $I_{z}^{(2)}$ increases. We expect that similar features exist also in the other stellar models.

\section{Conclusions}

Recent observations indicate precession of some pulsars. In order to understand the dynamics of these pulsars in detail, we have to know the moments of inertia, which play a crucial role in the Euler equation of motion. Motivated by this consideration, we have discussed the general relativistic effect on the moments of inertia of magnetically

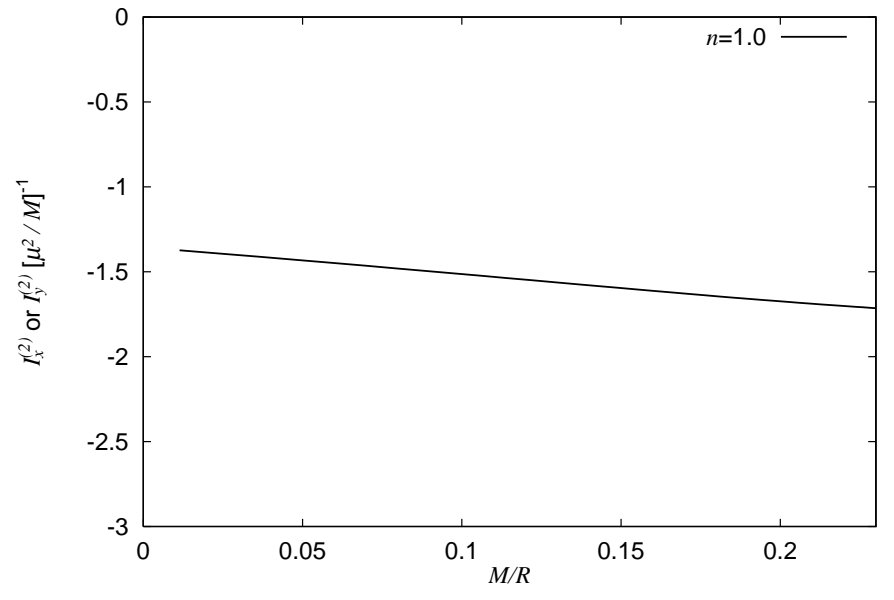

Fig. 3. The other components of the principal moments of inertia $I_{x}^{(2)}$ and $I_{y}^{(2)}$, which are derived for $n=1$.

deformed stars. By developing the formulation by Konno et al. (1999), we have considered the slow rotation of magnetically deformed stars on the symmetric axis in order to define the moment of inertia. The general expression for the magnetic correction $I_{z}^{(2)}$ to the moments of inertia of spherically symmetric stars was obtained, and the numerical estimates for various polytropic stellar models were also obtained. The other components of the moments of inertia $I_{x}^{(2)}$ and $I_{y}^{(2)}$ were discussed from the extension of relations in Newtonian gravity. From those results, we found that each principal moment of inertia is modified by a factor of 2 at most due to the general relativistic effect. Nevertheless, it seems that the general relativistic effect does not affect precessing motion noticeably in the case of free precession of pulsars. However, it is not clear whether the electromagnetic radiation reaction torque acting on the pulsars changes due to the general relativistic effect. If this torque is modified by the general relativistic effect, then the period of the wobbles would also be modified. Therefore, this point should be made clear in future investigation.

Acknowledgements. I would like to thank Y. Kojima for careful reading of the manuscript and for fruitful discussions and suggestions. I would also like to thank M. Kasai, H. Asada and $\mathrm{K}$. Ioka for valuable and useful comments, and M. Hosonuma for useful discussions. This work was supported in part by a Grant-in-Aid for Scientific Research Fellowship of the Ministry of Education, Science, Sports and Culture of Japan (No. 12001146).

\section{Appendix A: Summary of the perturbative approach}

We now review the perturbative approach developed by Konno et al. (1999) and Konno \& Kojima (2000). 


\section{A.1. Equations of order $\varepsilon_{B}$}

First, we consider the magnetic field described by $a_{\phi}$, which has the lowest order. This function obeys the following equation derived from the Maxwell equation,

$\mathrm{e}^{-\lambda} \frac{\mathrm{d}^{2} a_{\phi}}{\mathrm{d} r^{2}}+\frac{1}{2}\left(\nu^{\prime}-\lambda^{\prime}\right) \mathrm{e}^{-\lambda} \frac{\mathrm{d} a_{\phi}}{\mathrm{d} r}-\frac{2}{r^{2}} a_{\phi}=-4 \pi j_{\phi}$.

The electric current $j_{\phi}$ must satisfy the integrability condition (Ferraro 1954; Bonazzola et al. 1993)

$j_{\phi}=c_{0} r^{2}\left(\rho_{0}+p_{0}\right)$,

where $c_{0}$ is a constant of order $\varepsilon_{\mathrm{B}}$. This corresponds to the condition in which the star is in equilibrium. Equation (A.1) should be solved by imposing the condition in which $a_{\phi}$ vanishes at the stellar center and infinity and is connected smoothly at the stellar surface. The exterior solution can be obtained analytically (Ginzburg \& Ozernol 1965; Petterson 1974), while the interior solution was obtained numerically (Konno et al. 1999).

\section{A.2. Equations of order $\varepsilon_{B}^{2}$}

Next, we consider the effect of magnetic stress, which is of second order in $\varepsilon_{\mathrm{B}}$. This effect arises from the electromagnetic part of the stress-energy tensor (4). The second-order metric functions $h_{0}, h_{2}, m_{0}, m_{2}$ and $k_{2}$ can be obtained by solving the following two sets of differential equations and one algebraic equation derived from the Einstein equation $G^{\mu}{ }_{\nu}=8 \pi T_{\nu}^{\mu}$ and the equation of motion $T_{\nu ; \mu}^{\mu}=0$,

$$
\begin{aligned}
\frac{\mathrm{d} m_{0}}{\mathrm{~d} r}= & -4 \pi r^{2} \frac{\rho_{0}^{\prime}}{p_{0}^{\prime}}\left(\rho_{0}+p_{0}\right)\left(h_{0}-c_{1}\right) \\
& +\frac{1}{3} \mathrm{e}^{-\lambda}\left(a_{\phi}^{\prime}\right)^{2}+\frac{2}{3 r^{2}} a_{\phi}^{2}+\frac{8 \pi}{3} \frac{\rho_{0}^{\prime}}{p_{0}^{\prime}} a_{\phi} j_{\phi}, \\
\frac{\mathrm{d} h_{0}}{\mathrm{~d} r}= & \left(\frac{1}{r^{2}}+\frac{\nu^{\prime}}{r}\right) \mathrm{e}^{\lambda} m_{0}-4 \pi r \mathrm{e}^{\lambda}\left(\rho_{0}+p_{0}\right)\left(h_{0}-c_{1}\right) \\
& +\frac{1}{3 r}\left(a_{\phi}^{\prime}\right)^{2}-\frac{2}{3 r^{3}} \mathrm{e}^{\lambda} a_{\phi}^{2}+\frac{8 \pi}{3 r} \mathrm{e}^{\lambda} a_{\phi} j_{\phi}, \\
\frac{\mathrm{d} v_{2}}{\mathrm{~d} r}= & \left.-\nu^{\prime} h_{2}+\frac{2}{3} \mathrm{e}^{-\lambda}\left(\frac{1}{r}+\frac{\nu^{\prime}}{2}\right)\left(a_{\phi}^{\prime}\right)^{2}+\frac{4}{3 r^{2}} a_{\phi} a_{\phi}^{\prime}, \text { (A. (A) }\right) \\
\frac{\mathrm{d} h_{2}}{\mathrm{~d} r}= & -\frac{4 \mathrm{e}^{\lambda}}{r^{2} \nu^{\prime}} v_{2}+\left[8 \pi \frac{\mathrm{e}^{\lambda}}{\nu^{\prime}}\left(\rho_{0}+p_{0}\right)+\frac{2\left(1-\mathrm{e}^{\lambda}\right)}{r^{2} \nu^{\prime}}-\nu^{\prime}\right] h_{2} \\
& +\frac{8}{3 r^{4} \nu^{\prime}} \mathrm{e}^{\lambda} a_{\phi}^{2}+\frac{8}{3 r^{3} \nu^{\prime}}\left(1+\frac{r \nu^{\prime}}{2}\right) a_{\phi} a_{\phi}^{\prime} \\
& +\left(\frac{1}{3} \nu^{\prime} \mathrm{e}^{-\lambda}+\frac{2}{3 r^{2} \nu^{\prime}}\right)\left(a_{\phi}^{\prime}\right)^{2}+\frac{16 \pi}{3 r^{2} \nu^{\prime}} \mathrm{e}^{\lambda} a_{\phi} j_{\phi}, \text { (A.6) } \\
m_{2}= & r \mathrm{e}^{-\lambda}\left[-h_{2}+\frac{2}{3} \mathrm{e}^{-\lambda}\left(a_{\phi}^{\prime}\right)^{2}\right],
\end{aligned}
$$

where $c_{1}$ is a constant fixed by the junction condition at the stellar surface, and $v_{2}$ is defined as $v_{2}=h_{2}+k_{2}$. The differential Eqs. (A.3)-(A.6) should be solved by imposing the boundary conditions

$m_{0}, h_{2}, k_{2} \rightarrow 0, \quad h_{0} \rightarrow$ const. as $\quad r \rightarrow 0$,

$$
h_{0}, h_{2} \rightarrow 0, \quad m_{0} \rightarrow \text { const. }, \quad k_{2} \rightarrow \frac{1}{r^{\alpha}}(\alpha \geq 3)
$$

$$
\text { as } r \rightarrow \infty \text {. }
$$

Here, note that the value of $m_{0}$ at infinity corresponds to a mass shift associated with the perturbation. Furthermore, the junction condition at the stellar surface is written as (O'Brien \& Synge 1952)

$$
\begin{gathered}
\left.g_{\mu \nu}\right|_{+\xi}=\left.g_{\mu \nu}\right|_{-\xi} \quad(\mu, \nu=t, r, \theta, \phi), \\
\left.g_{\lambda \sigma, r}\right|_{+\xi}=\left.g_{\lambda \sigma, r}\right|_{-\xi} \quad(\lambda, \sigma=t, \theta, \phi),
\end{gathered}
$$

where $\xi$ denotes the boundary surface of the star.

Once we have the above metric functions, we can derive the second-order corrections of pressure and density from the relations

$$
\begin{aligned}
& p_{20}=-\left(\rho_{0}+p_{0}\right) h_{0}+\frac{2}{3 r^{2}} a_{\phi} j_{\phi}+c_{1}\left(\rho_{0}+p_{0}\right) \\
& p_{22}=-\left(\rho_{0}+p_{0}\right) h_{2}-\frac{2}{3 r^{2}} a_{\phi} j_{\phi} \\
& \rho_{20}=\frac{\rho_{0}^{\prime}}{p_{0}^{\prime}} p_{20} \\
& \rho_{22}=\frac{\rho_{0}^{\prime}}{p_{0}^{\prime}} p_{22}
\end{aligned}
$$

Therefore, the stellar structure which is subject to magnetic deformation can be determined by solving Eqs. (A.3)-(A.6). The exterior solution for the metric functions was obtained analytically, while the interior solution was calculated numerically by Konno et al. (1999). The fact that the evaluation of ellipticity using the results of the metric functions reproduced the Newtonian result derived by Ferraro (1954) in the Newtonian limit (see Konno et al. 2000) justifies this formalism.

\section{A.3. Equations of order $\varepsilon_{\Omega} \varepsilon_{B}$}

Finally, we consider the quantities $a_{t 0}$ and $a_{t 2}$ of order $\varepsilon_{\mathrm{B}} \varepsilon_{\Omega}$, which are related to the electric fields induced by stellar rotation. These functions obey the Maxwell equation. Outside the star, we have

$$
\begin{aligned}
& \mathrm{e}^{-\lambda} \frac{\mathrm{d}^{2} a_{t 0}}{\mathrm{~d} r^{2}}+\frac{2 \mathrm{e}^{-\lambda}}{r} \frac{\mathrm{d} a_{t 0}}{\mathrm{~d} r} \\
& \quad=\frac{2}{3}\left[\left(\lambda^{\prime}+\frac{2}{r}\right) \omega+\omega^{\prime}\right] \mathrm{e}^{-\lambda} a_{\phi}^{\prime}+\frac{4}{3 r^{2}} \omega a_{\phi}, \\
& \mathrm{e}^{-\lambda} \frac{\mathrm{d}^{2} a_{t 2}}{\mathrm{~d} r^{2}}+\frac{2 \mathrm{e}^{-\lambda}}{r} \frac{\mathrm{d} a_{t 2}}{\mathrm{~d} r}-\frac{6}{r^{2}} a_{t 2} \\
& \quad=-\frac{2}{3}\left[\left(\lambda^{\prime}+\frac{2}{r}\right) \omega+\omega^{\prime}\right] \mathrm{e}^{-\lambda} a_{\phi}^{\prime}+\frac{8}{3 r^{2}} \omega a_{\phi}
\end{aligned}
$$

where the function $\omega$ of first order in $\varepsilon_{\Omega}$ was discussed in detail by Hartle (1967). These differential equations can also be solved analytically (see Muslimov \& Tsygan 1986; Konno \& Kojima 2000 for the detailed calculations). Inside the star, the assumption of a perfectly conducting interior yields the expressions (Bonazzola et al. 1993; 
Bocquet et al. 1995),

$a_{t 0}=\frac{2}{3} \Omega a_{\phi}+c_{2}$,

$a_{t 2}=-\frac{2}{3} \Omega a_{\phi}$

where $c_{2}$ is a constant fixed by the junction condition at the stellar surface. In this case, $a_{t 0}$ and $a_{t 2}$ are not connected smoothly at the stellar surface, owing to the induced surface charge.

\section{References}

Baykal, A., \& Swank, J. 1996, ApJ, 460, 470

Baykal, A., Swank, J. H., Strohmayer, T., \& Stark, M. J. 1998, A\&A, 336, 173

Bertotti, B., \& Anile, A. M. 1973, A\&A, 28, 429.

Bocquet, M., Bonazzola, S., Gourgoulhon, E., \& Novak, J. 1995, A\&A, 301, 757

Bonazzola, S., Gourgoulhon, E., Salgado, M., \& Marck, J. A. 1993, A\&A, 278, 421

Chandrasekhar, S., \& Miller, J. C. 1974, MNRAS, 167, 63

Cutler, C., \& Jones, D. I. 2000, preprint [gr-qc/0008021]

D'Alessandro, F., McCulloch, P. M., Hamilton, P. A., \& Deshpande, A. A. 1995, MNRAS, 277, 1033

Davis, L., \& Goldstein, M. 1970, ApJ, 159, L81

Duncan, R. C., \& Thompson, C. 1992, ApJ, 392, L9

Ferraro, V. C. A. 1954, ApJ, 119, 407

Geroch, R. 1970, J. Math. Phys., 11, 2580

Ginzburg, V. L., \& Ozernol, L. M. 1965, Sov. Phys. JETP, 20, 689
Goldreich, P. 1970, ApJ, 160, L11

Hansen, R. O. 1974, J. Math. Phys., 15, 46

Hartle, J. B. 1967, ApJ, 150, 1005

Kojima, Y., \& Hosonuma, M. 1999, ApJ, 520, 788

Konno, K., \& Kojima, Y. 2000, Prog. Theor. Phys., 104, 1117

Konno, K., Obata, T., \& Kojima, Y. 1999, A\&A, 352, 211

Konno, K., Obata, T., \& Kojima, Y. 2000, A\&A, 356, 234

Kouveliotou, C., Dieters, S., Strohmayer, T., et al. 1998, Nature, 393, 235

Kouveliotou, C., Strohmayer, T., Hurley, K., et al. 1999, ApJ, 510, L115

Melatos, A. 1999, ApJ, 519, L77

Melatos, A. 2000, MNRAS, 313, 217

Mereghetti, S. 1995, ApJ, 455, 598

Mereghetti, S., \& Stela, L. 1995, ApJ, 442, L17

Mészáros, P. 1992, High-Energy Radiation from Magnetized Neutron Stars (The University of Chicago Press, USA)

Muslimov, A. G., \& Tsygan, A. I. 1986, SvA, 30, 567

O'Brien, S., \& Synge, J. L. 1952, Comm. Dublin Inst. Advanced Studies, A no. 9

Oosterbroek, T., Parmar, A. N., Mereghetti, S., \& Israel, G. L. 1998, A\&A, 334, 925

Petterson, J. A. 1974, Phys. Rev. D, 10, 3166

Quintana, H. 1976, ApJ, 207, 279

Ravenhall, D. G., \& Pethick, C. J. 1994, ApJ, 424, 846

Shemar, S. L., \& Lyne, A. G. 1996, MNRAS, 282, 677

Stairs, I. H., Lyne, A. G., \& Shemar, S. L. 2000, Nature, 406, 484

Thompson, C., \& Duncan, R. C. 1993, ApJ, 408, 194

Thompson, C., \& Duncan, R. C. 1995, MNRAS, 275, 255

Thompson, C., \& Duncan, R. C. 1996, ApJ, 473, 322

Wang, N., Manchester, R. N., Pace, R. T., et al. 2000, MNRAS, 317,843 